\title{
How should the Clinician Behave in the Controversy between Metformin and Vitamin B12 Subclinical Nutritional Deficiency?
}

\section{Belazzouz Abderrahmen Youssouf*}

Sisters Bedj hospital, Internal medicine department, Chlef, Algeria

*Corresponding Author: Belazzouz Abderrahmen Youssouf, Sisters Bedj hospital, Internal medicine department, Chlef, Algeria.

Received: October 30, 2019; Published: November 06, 2019

DOI: 10.31080/ASNH.2019.03.0533

\begin{abstract}
In recent days, a nutritional complication is more and more reported in patients with type 2 diabetes under metformin represented by the infra clinical deficit in vitamin B12 defined by a normal or low level of vitamin B12 and a moderate rise in its intracellular cofactors such as: homocysteine and Holotranscobalamin.

The prevalence of this deficiency among type 2 diabetics under metformin varies from one series to another. Several factors have been implicated in this deficit, such as advanced age, ethnic origin and vegetarian diet. Nevertheless a dose beyond 2 grams per day of metformin and a duration of treatment beyond 10 years also seem to be involved.

The pathophysiological mechanism implicated seems to be complex by an intestinal calcium depletion secondary to metformin intake but independently of its digestive side effects (such as diarrhea, vomiting). Studies have shown that a calcium intake of 1 gram per day improves the level of intracellular cofactors of vitamin B12 without causing a change in vitamin B12 status.

Other ways of supplementation exist by vitamin B12 (oral and injectable). Suspension of treatment with metformin is never to be considered as a therapeutic approach view these benefits especially as improving insulin sensitivity metformin allows good diffusion of vitamin B12 and suitable intracellular action.

Diabetic patients on metformin can benefit from an effective therapeutic dose allowing a good glycemic balance subject to periodic screening for vitamin B12 deficiency and a good nutritional intake of vitamin B12 in case of deficiency or preventive calcium intake in the patients at risk of deficit.
\end{abstract}

Keywords: Vitamin B12; Metformin

\section{Introduction}

By dint of submitting patients with type 2 diabetes under sometimes drastic regime imposed by a glycemic imbalance or diabetic nephropathy or recently for the purpose of a reversibility of the disease, it was necessary to periodically monitor their nutritional status and especially check their vitamins.

The iatrogenic origin in this nutritional deficiency has been strongly incriminated not only through the diet but also through oral antidiabetic drugs such as metformin which is the oldest oral antidiabetic with which we have the most cardiovascular safety.
However, recent studies have shown that even sulfonamides participate in this deficiency and patients on metformin alone have less deficit compared to diabetics on metformin in combination with sulfonamide [1].

Nevertheless, the largest series concerned the metformin molecule, and the prevalence of this deficiency under metformin remains variable [2]. This is partly due to the variability in the criteria for defining vitamin B12 deficiency. 


\section{Development}

The subclinical deficit of vitamin B12 is defined clinically by the absence of hematological or neurological signs, biochemically by a level of vitamin B12 (119-186) pmol/l; and its cofactors such as: homocysteine (13.6-19.2) umol/l; holotranscobalamin (20-37) pmol/l; MMA: Methyl malonylacid (0.35-0.84) umol/l [3].

The pathophysiological mechanism of metformin-related vitamin B12 subclinical deficiency is intestinal calcium depletion, since calcium is essential for the absorption of vitamin B12 [4].

Others studies have shown that daily supplementation with 1gram calcium per day seems sufficient to reduce intracellular cofactors of vitamin B12 without any change in the level of this vitamin. As well as the non-elevated vitamin B12 level reflects its action as well as its consumption [5].

Vitamin B12 supplementation, whether oral or injectable, is effective [6] nevertheless it remains a symptomatic treatment and the ideal remains to treat the etiology which can be either small dietary modifications allowing at the same time the non-aggravation of the glycemic imbalance or Diabetic nephropathy as well as the preservation of adequate vitamin status (nutritional tips proposed by dieticians to protect sources of B12 vitamin that does not alter the glycemic and protein balance) [7]. In fact, dietetics is an art that makes it possible to establish a balance between the desired objective through this diet and the non-deterioration of the existing vitamin stock.

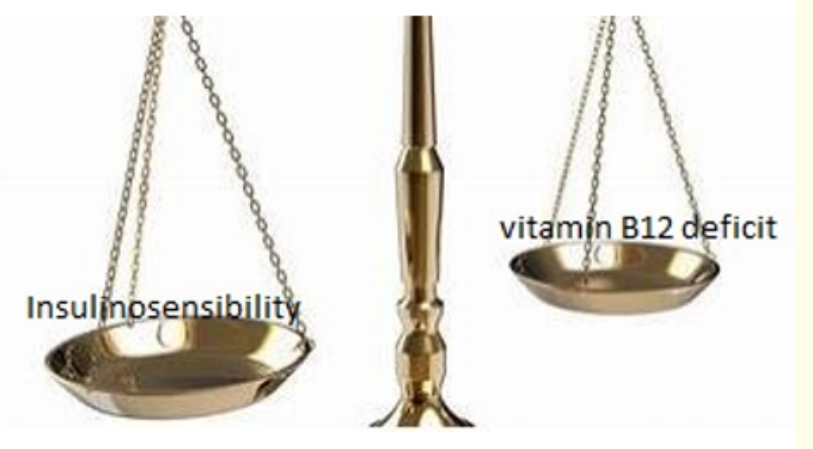

\section{Conclusion}

Metformin remains the molecule of choice in diabetes type 2 for its multiple benefits and its prescription must be accompanied by a suitable monitoring of vitamin B12 status, especially in patients at risk with adequate calcium intake in this patient population.

\section{Bibliography}

1. Marwan Awad Ahmed. "Metformin and Vitamin B12 Deficiency: Where Do We Stand?" Journal of Pharmaceutical Sciences 19.3 (2016): 382-396.

2. Donghoon Kang., et al. "Higher Prevalence of Metformin-Induced Vitamin B12 Deficiency in Sulfonylurea Combination Compared with Insulin Combination in Patients with Type 2 Diabetes: A Cross-Sectional Study". PLoS One 9.10 (2014): e109878.

3. Luciana Hannibal., et al. "Biomarkers and Algorithms for the Diagnosis of Vitamin B12 Deficiency". Frontiers in Molecular Biosciences 3 (2016): 1-27.

4. Herbert V and Castle WB. "Divalent cation and pH dependence of rat intrinsic factor action in everted sacs and mucosal homogenates of rat small intestine". Journal of Clinical Investigation 40 (1961): 1978-1983.

5. WA Bauman., et al. "Increased intake of calcium reverses vitamin B12 malabsorption induced by metformin". Diabetes Care 23.9 (2000): 1227-1231.

6. Bor M., et al. "A daily intake of approximately 6 microg vitamin B-12 appears to saturate all the vitamin B-12-related variables in Danish postmenopausal women". American Journal of Clinical Nutrition 83 (2006): 52-58.

7. Canadian Nutrient File (2015).

Volume 3 Issue 12 December 2019 (c) All rights are reserved by Belazzouz Abderrahmen Youssouf.

Figure 